\title{
COMPORTAMIENTO DE FORRAJEO EN NUTRIAS \\ GIGANTES (Pteronura brasiliensis), UN ESTUDIO DE CASO EN SANTA ROSA DE SAPUARA, DEPARTAMENTO DE GUAINÍA, COLOMBIA
}

\author{
FORAGING BEHAVIOR IN GIANT OTTERS (Pteronura brasiliensis), \\ A CASE STUDY IN SANTA ROSA DE SAPUARA, DEPARTMENT OF \\ GUAINÍA, COLOMBIA.
}

\section{${ }^{1}$ Engelbert Chavez-Fontecha, 2 Myriam Janeth Ortega Torres, 3 Jose Camilo Torres Romero}

${ }^{1}$ Lic Biología, Candidato a Msc U Externado de Colombia Der Adtivo.

2 MSc Producción animal, estudiante doctorado Biología Molecular y Biomedicas- Universidad del País Vasco- UNAD.

${ }^{3}$ Doctor en Bioquímica. UNAD.

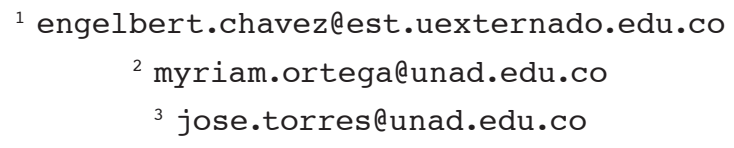

\section{RESUMEN}

El comportamiento alimenticio en la nutria gigante (Pteronura brasiliensis) ha sido descrito por varios autores, sin embargo, la manera coordinada en que la nutria participa en la caza y obtiene su alimentación, aún no se ha estudiado en detalle. Este trabajo sistematiza la información de observaciones realizadas sobre el comportamiento de caza de Pteronura brasiliensis, en la laguna del Sapuara en el departamento de Guanía, Colombia, y permitió describir la caza como un comportamiento cooperativo y altamente organizado. Se realizó un estudio del comportamiento de caza a través de observaciones ad-libitum, desde rocas y árboles de 10 a 20 metros de altura con un rango de visión horizontal de $110^{\circ}$, los parámetros analizados fueron: estrategia de caza, tipo de orilla, tiempo invertido en la caza, número de nutrias activas (nutrias que participaron en la caza), profundidad, condiciones climáticas, y efectividad de la caza. En total se observaron 11 comportamientos cooperativos de caza distintos, los cuales se pudieron agrupar en tres grupos: Punta de flecha, filas y corraleo. El más frecuente de todos los observados en faenas de cacería fue punta de flecha con un porcentaje de $38 \%$, con un número promedio de 7 nutrias participando de 
la caza y con un porcentaje de éxito de hasta el $87 \%$. Este es un nuevo instrumento para abordar el estudio del comportamiento de la nutria gigante desde la perspectiva cooperativa de caza.

Palabras clave: Pteronura, forrajeo, cooperación comportamiento y Guainía-Colombía.

\section{ABSTRACT}

The feeding behavior in the giant otter (Pteronura brasiliensis) has been described by several authors, however, the coordinated way in which the otter takes part in the hunting and obtains his feeding, has not been studied in detail. This paper systematizes the information of observations on the hunting behavior of Pteronura brasiliensis, in the Sapuara lagoon in the department of Guanía, Colombia, and allowed to describe hunting as a cooperative and highly organized behavior. This behavior has been studied through ad-libitum observations, from rocks and trees of 10 to 20 meters high with a horizontal viewing range of $110^{\circ}$. Some of the parameters analyzed were fishing strategy, type of shore, time spent on fishing, number of active otters (otters that participated in the fishing), depth, climatic conditions, and effectiveness of hunting. In the end, 11 cooperative fishing behaviors were observed, which were classified into three groups: Arrowhead, ranks and rounding. The most frequent of all those observed in farm work was arrowhead with a percentage of $38 \%$, with an average number of 7 otters participating in the hunt and with a success rate of up to $87 \%$. This is a new instrument for the approach of the study of the behavior of $P$. brasiliensis from the cooperative perspective of hunting.

Key words: Pteronura, fishing, cooperation behavior and Guainía-Colombía

\section{INTRODUCCIÓN}

El desarrollo de la sociabilidad entre las especies ha sido determinado por la relación costo beneficio (Alexander 1974). El establecimiento de grupos sociales entre animales incrementa la competencia intraespecífica por recursos o posibilidades reproductivas (Alexander 1974; Blundell, Ben-David y Browyer 2002) (Blundell, Ben-David, Groves, Browyer, y Geffen 2004). Considerando que pueden presentar beneficios como la evasión de depredadores, defensa de territorio, obtención de alimento, entre otros (Alcock, 1993) (Blundell, Ben-David, y Bowyer, 2002).

La organización de animales desde la perspectiva cooperativa es una estrategia que ha generado condiciones favorables para la supervivencia de especies a lo largo de la evolución, especialmente en carnívoros (Kruuk 2006).
Dentro de los comportamientos sociales en las nutrias se describen individuos solitarios (Lutra lutra en ambientes marinos) (Kruuk y Moorhouse, 1991); pares monógamos (Lontra felina, Ostfeld et al., 1989); reuniones familiares (Lutra maculicollis) con variación interespecífica de la socialidad grupal; grupo familiar (Lontra canadensis, Melquist y Hornocker, 1983), y Aonyx capensis (Arden-Clarke 1986); y familias extendidas (Pteronura brasiliensis, Kruuk 2006).

Pteronura brasiliensis, comúnmente conocida como nutria gigante, es un mamífero, semiacuático especializado y gregario (Kruuk, 1995 y Álvarez-León, 2009), y como otros grupos sociales como los cánidos (Creel \& Creel, 1995 y Bohórquez, 1994), presenta una organización social definida con una pareja alfa 
(Evangelista, 2004) y una vinculación social de individuos genéticamente interrelacionados. La nutria gigante es una especie oportunista (Gómez, 2004), que permite mantener una gran diversidad de especies de peces (Velandia y Vásquez, 2015). Cuando ellos cazan, buscan individuos menos sanos y fáciles de atrapar, que mejoran y equilibran las poblaciones de los peces que se alimentan (Portocarrero, Morales, Díaz y Millán, 2009). En nutrias en cautiverio, se demostró que las nutrias eligen sus presas por su vulnerabilidad (Mason \& Macdonald, 2009).

Por lo anterior, y evidenciando que la viabilidad del alimento no es un estado o condición fija en la naturaleza, sino presente como un fenómeno dinámico que depende de muchos factores ambientales individuales e interconectados, se propuso como objetivo en el desarrollo de esta investigación establecer si el número de individuos en una población de nutrias gigantes (Ptenoura brasiliensis) tiene relación con las actividades de forrajeo, y si las nutrias presentan comportamientos cooperativos para desarrollarlas, además de establecer cómo estas influyen en la eficiencia al obtener alimentos, partiendo de "comprender la relación entre las nutrias y su alimento" (Kruuk, 2006) y de ello "considerar interacciones reales entre nutria y pez" (Kruuk, 2006).

Uno de los alcances del presente artículo es proporcionar nuevos elementos a la interacción antes mencionada, que permitan mejorar la gestión y conservación de los hábitats naturales de esta especie actualmente en peligro (EN) por la UICN (Groenendijk, Duplaix, Marmontel, Van Damme \& Schenck, C. 2015; Trujillo; Botello \& Carrasquilla 2006), ya que la nutria gigante interactúa con los humanos y mantiene la diversidad íctica (Velandia \&
Vásquez, 2015) importante para el bienestar de las poblaciones humanas con las que comparten los ecosistemas. Por otro lado, esta primera aproximación al comportamiento de Pteronura brasiliensis desde la perspectiva cooperativa de caza se convierte en un nuevo instrumento para abordar el estudio del grupo.

\section{MATERIALES Y MÉTODOS}

\section{Área de estudio}

La investigación se realizó en la jurisdicción del corregimiento de Barrancominas, en el departamento de Guainía, ubicado en el sureste de Colombia, entre el río Guaviare y la República Federal de Brasil (Rudas y Prieto, 1998), en la laguna de Santa Rosa de Sapua-

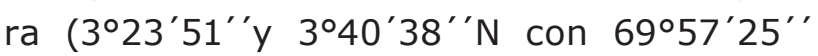
y $69^{\circ} 20^{\prime} 57^{\prime \prime} \mathrm{W}$ ) (Figura. 1). La ecogeografía del área de estudio pertenece a la selva transicional del Guainía, que se puede encontrar entre los ríos Vichada e Inírida (Domínguez, 1985), con temperaturas que oscilan entre $22^{\circ} \mathrm{C}$ y $40^{\circ} \mathrm{C}$. Su régimen de lluvias es biestacional con cerca de 2,800 mm, siendo los meses lluviosos de abril a septiembre y los meses secos de octubre a marzo (IDEAM, 2012) (Pérez, Bucheli \& Giraldo, 2005). En la zona de influencia de estudio se presentan comunidades campesinas e indígenas de puinaves, sikuanis y paipocos principalmente, con una alta dependencia de los recursos naturales especialmente de la pesca, cacería y explotación de suelos asociados a las vegas del rio Guaviare para para autoconsumo y una menor proporción comercialización. En la actualidad hacia las ciudades de Villavicencio, departamento del Meta, y Bogotá D.C., del área de influencia de la zona de estudio se explotan cerca de 200 toneladas anuales de pescado, como también cacao silvestre, artesanías, entre otras. 


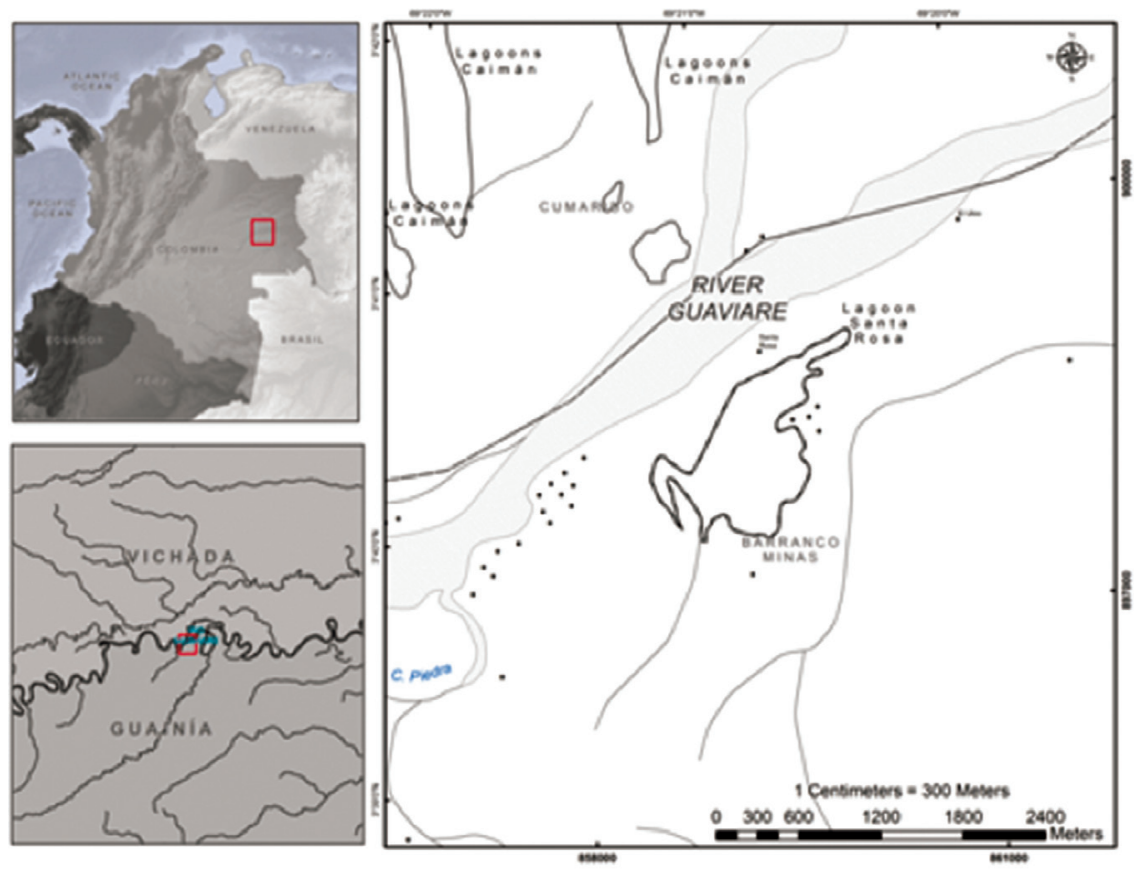

Figura 1. Zona de estudio.

\section{Observaciones}

Las observaciones de campo se llevaron a cabo durante dos fases, el primero de marzo de 2007 a enero de 2008, con un tiempo de esfuerzo total de 297 horas. El segundo, entre septiembre de 2012 hasta enero de 2013 y noviembre de 2015 , con un tiempo de esfuerzo total de 310 horas. Los datos de comportamiento se realizaron con observaciones ad libitum (Martin y Bateson, 1991; Altmann, 1974). Con base en estas observaciones se analizó, el éxito en la caza (efectivo: éxito de caza individual), el número de nutrias involucradas en la caza y sus roles, el horario de caza y la descripción de la alimentación cooperativa de la nutria gigante. Las observaciones se hicieron entre las 6:00 a.m. y las 11:00 a.m., y entre las 2:00 p.m. y las 5:00 p.m, horas establecidas en la fase preliminar de campo, como de mayor actividad del grupo.

El seguimiento a la especie se realizó desde una canoa de $5 \mathrm{~m}$ de largo y 0,4 $\mathrm{m}$ de ancho, impulsada por remo, y desde 8 puntos altos como troncos caídos, árboles fáciles de trepar y desde la orilla de la laguna en los afloramientos rocosos con más de $15 \mathrm{~m}$ de altura, con un ángulo de campo visual superior a los $110^{\circ}$ y por lo menos un ángulo de $10^{\circ}$ con respecto a la horizontal. Estos puntos de observación fueron definidos a partir de un estudio previo realizado en la zona que permitió establecer las áreas de interés en tierra y agua, comportamientos generales del grupo y uso de hábitat. La profundidad se determinó mediante regla calibrada con plomada y el promedio de tres mediciones en cada punto, en las áreas de interés.

Este estudio se realizó en animales silvestres, teniendo en cuenta un mínimo de 50 m de distancia del grupo nutria para no interferir con las actividades de la especie (Mumm, Urrutia \& Knörnschild, 2014). De esta manera, el monitoreo desde los puntos de observación se complementó con registros fotográficos utilizando binoculares (Tasco Futura 35x55 mm) y cámaras digitales (lente zoom macro Canon Rebel XT 125-300 una lente Sigma 2x HD y lente macro zoom NIKON 5100 125-300). 
La inmersión de forrajeo se calculó utilizando el tiempo de inmersión de cada individuo y la efectividad de la caza, con apoyo en un cronómetro (Casio Hs30w1 / 100 LapMemory 10). La efectividad de la caza se estimó a partir de individuos (identificación de individuos por su marca única de garganta) que obtuvieron presa con respecto al número total de individuos activos en la caza (individuos que participan en la caza).

\section{Análisis estadístico}

La estadística descriptiva para cada parámetro analizado se obtuvo utilizando el software $\mathrm{R}$ y los resultados se analizaron usando el modelo estadístico Chi-cuadrado de Pearson con $\mathrm{n}=45$ (siendo $\mathrm{n}$ el número de eventos en los que el desarrollo de la cooperativa de caza era evidente). Este análisis se realizó con el software $R$ ( $R$ versión 3.0.3. (2014-03-06)), para establecer la asociación y correlaciones de variables de alimentación cooperativa (punta de flecha, filas y corraleo), lugar donde se realizó la caza (vegetación sumergida, frente a la costa y aguas abiertas), tiempo invertido en la caza, número de participantes nutrias activas (individuos involucrados en la caza), profundidad de la zona de caza, estación climática (invierno y estación seca) y caza efectiva.

\section{RESULTADOS Y DISCUSIÓN}

El estudio se desarrolló en dos fases. En la primera fase, el tiempo de contacto alcanzado de la visualización directa de la especie durante las actividades de caza fue de 1.491 minutos y en la segunda fase de 1.150 minutos. La familia de nutrias gigantes de la laguna de estudio estaba conformada por 2 adultos, 3 subadultos, 2 juveniles y 2 crías para la primera fase de campo (9 en total), en la segunda fase se observó un subadulto adicional sin crías (8 en total).

En cuanto al número e integrantes de grupos o familias de las nutrias gigantes existe consenso con la mayoría de los autores. Evangelista \& Rosas (2011) observaron ochenta individuos en Brasil, de las cuales el $88.7 \%$ fueron encontradas en 15 grupos de nutrias, siendo el mayor número de individuos un grupo de 9 animales y con un tamaño promedio de 4,46 individuos (Evangelista \& Rosas, 2011. pág. 33). Carrasquilla \& Trujillo (2004) precisaron en nutrias del Rio Orinoco que estos grupos están conformados por una pareja estable (alfa) y una o dos generaciones de hijos (subadultos o juveniles) con tres a nueve individuos (Carrasquilla \& Trujillo, 2004, pág. 181). Los hallazgos realizados en la presente investigación concuerdan con el número y características de los integrantes de los grupos de nutrias estudiados por Carrasquilla \& Trujillo (2004) y Evangelista \& Rosas (2011), al corroborar que la familia de Pteronura brasiliensis en la laguna de estudio, está conformada por dos adultos, tres subadultos, dos juveniles y dos crías en la primera fase ( 9 individuos) y en la segunda fase hasta tres juveniles sin crías (8 individuos).

Uno de los aspectos relevantes en el comportamiento cooperativo es el liderazgo que realiza la pareja alfa, debido a que son estos quienes tienen la experiencia generacional y memoria grupal (Davenport, 2010), que permite el desarrollo efectivo de la caza. La anterior definición de roles robustece el hallazgo de la caza cooperativa a partir de la ejecución de alternativas de captura para la obtención del alimento en nutrias gigantes, haciéndose evidente que la familia de nutrias gigantes de la zona de estudio tiene un alto comportamiento gregario (Kruuk, 2006,) y sus actividades de caza también se rigen por dicho comportamiento de cooperación (Portocarrero et al., 2009).

Se identificó que la pareja alfa lidera los ejercicios de caza, esto se determina porque el macho y la hembra, son quienes siempre van de primeros en la punta del grupo emitiendo 
sonidos de forma constante. El macho fue identificado por el tamaño y comportamiento en el momento de cortejo. Se estableció a partir del análisis de número de individuos en el ejercicio de caza, que el tamaño para el desarrollo de la mayoría de las estrategias es $\bar{X}=7$ (Figura.2), siendo este número de individuos importante en el desarrollo de las actividades de caza y comportamientos sociales como la niñera, para el cuidado parental y defensa del territorio entre otros, al igual que en el $93 \%$ de las estrategias de caza identificadas en la laguna de Santa Rosa de Sapuara, el macho y la hembra alfa lideran las actividades caza.

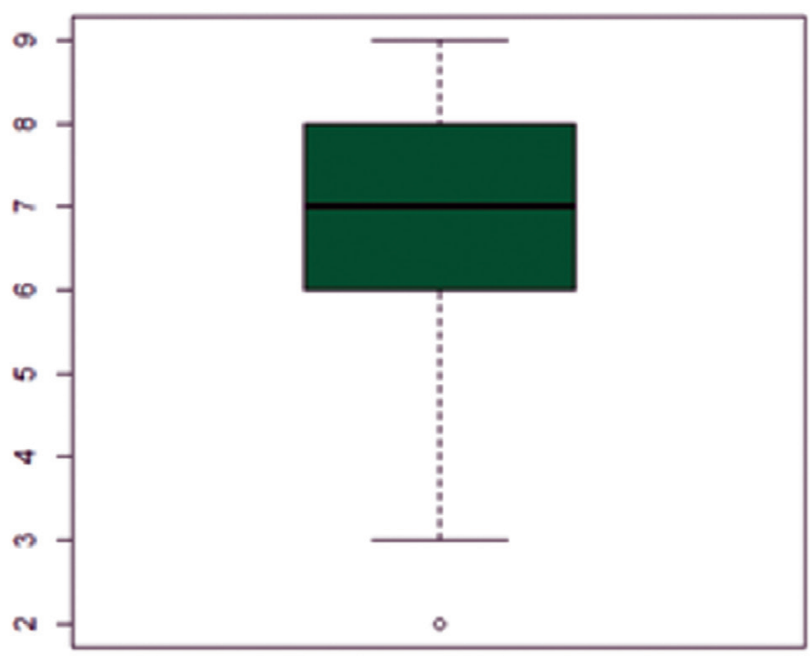

Figura 2. Número de individuos en la caza.

Se evidenció que las crías de nutrias participan en algunas de las actividades de caza, siendo este fenómeno parte de las actividades de aprendizaje a partir de la imitación de algunas conductas de los tutores. El aprendizaje por imitación es reportado por Kruuk (2006) en nutrias, Whiten et al., (1996) en primates, y Maier (2001) en otros ordenes. Este comportamiento social corresponde a especies zoológicas de vida gregaria, cuya estructura determina o prescribe las relaciones de cada individuo al interior del grupo, y con ello establece una organización que permite a cada uno de los individuos asignarse su lugar dentro del grupo en que se encuentra (Klopfer, 1976) (Figura. 3).

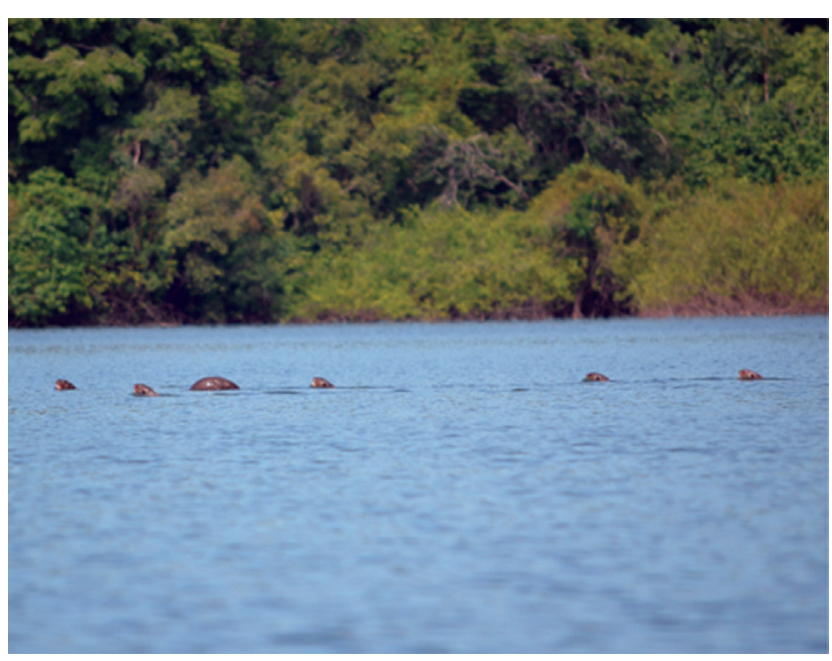

Figura 3. Grupo de caza en aguas abiertas. Velandia C.

Se encontró que la nutria gigante tiene comportamientos cooperativos de forrajeo $(n=45)$, donde las estrategias y el área de caza tienen asociación, encontrando un p-valor $=0$ con un nivel de confianza del $90 \%$. Estas estrategias se agruparon de acuerdo a la forma de organización del grupo al momento de forrajear, en tres tipos:

1. Punta de flecha: consiste en la disposición en forma escalonada y triangular de cada uno de los individuos participantes en la práctica, estructura similar a la punta de una flecha (Figura. 4), con una recurrencia del $38 \%$. 


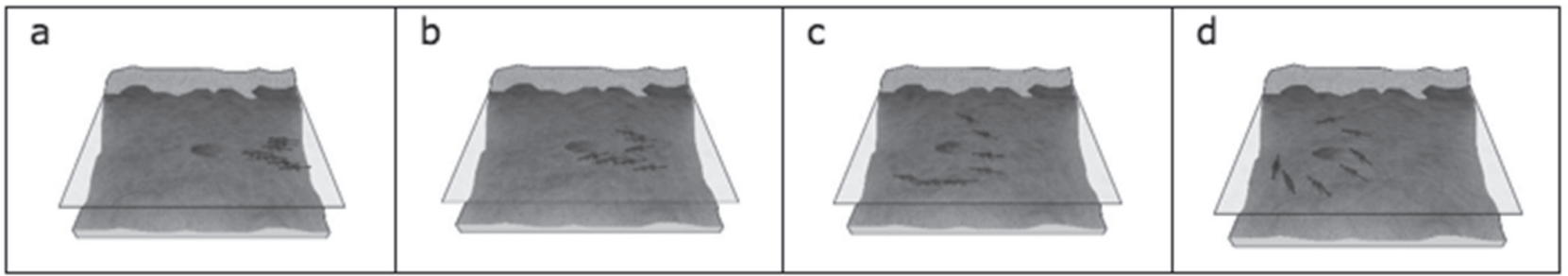

Punta Flecha en aguas abiertas.

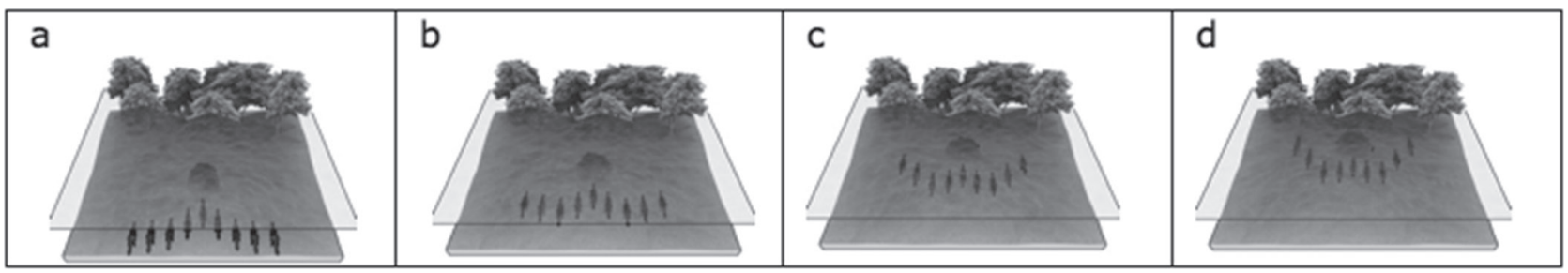

Punta Flecha contra vegetación sumergida.

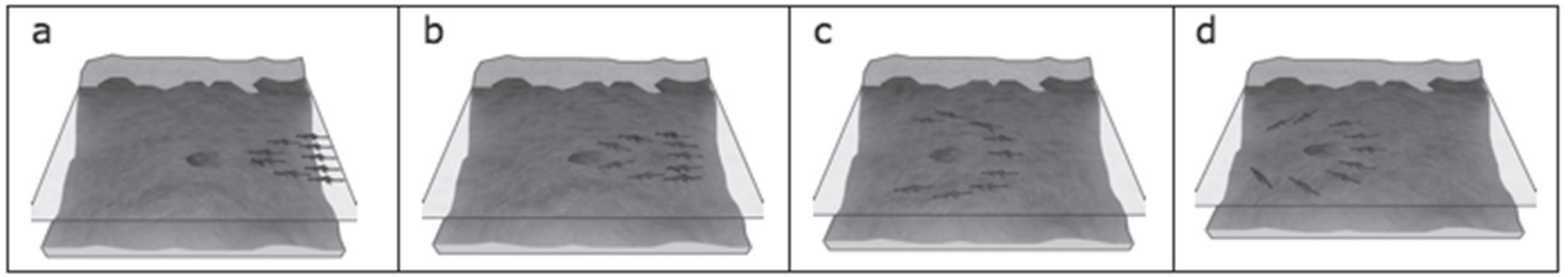

Punta de Flecha 2-2-5, en aguas abiertas.

Figura 4. Forrajeo en Punta de Flecha.

2. Filas: las nutrias se agrupan en líneas paralelas ejerciendo control del cardumen (Figura. 5), en $33 \%$ de las observaciones realizadas.

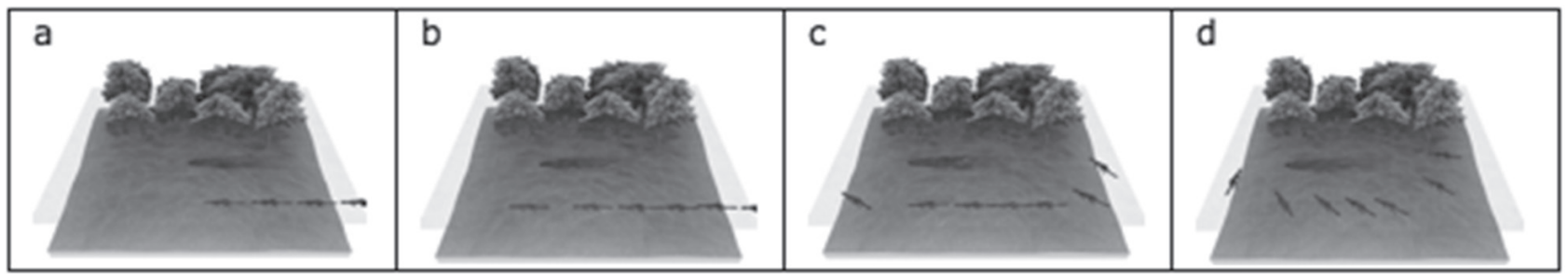

Fila paralela a la orilla contra vegetación sumergida.

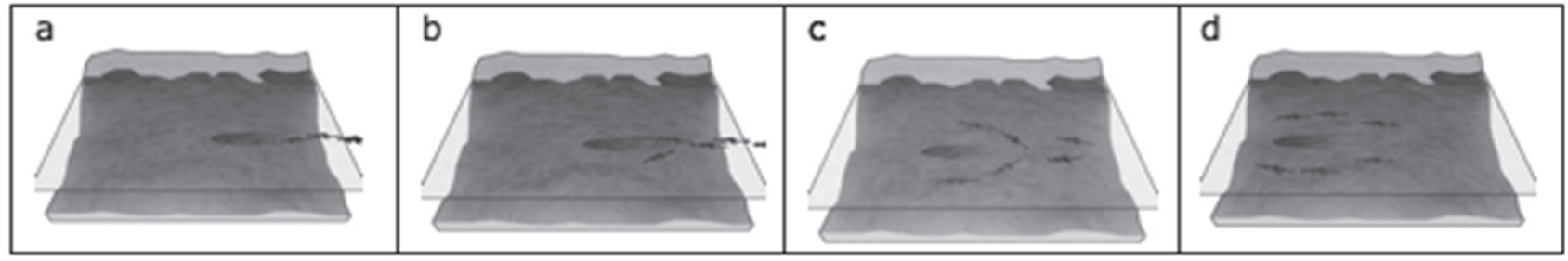

Estrategia de cerramiento en " $Y$ " en aguas abiertas 


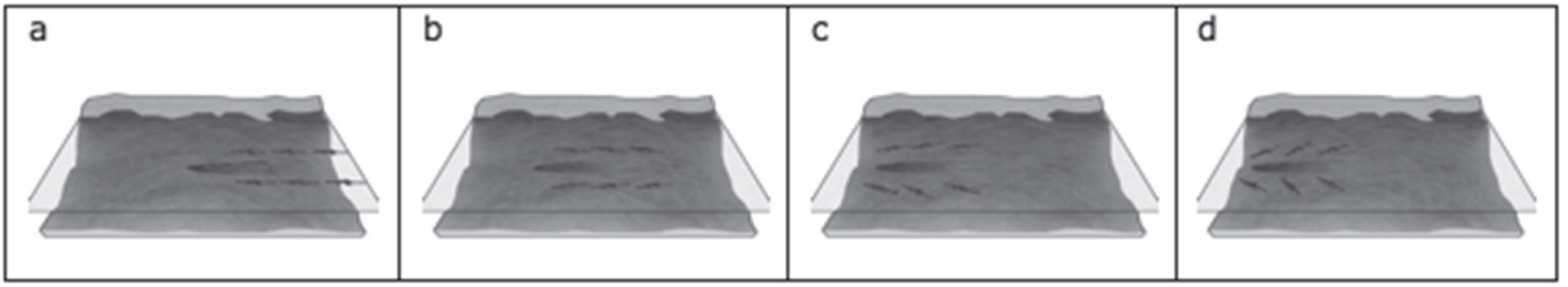

Estrategia de cerramiento con filas paralelas.

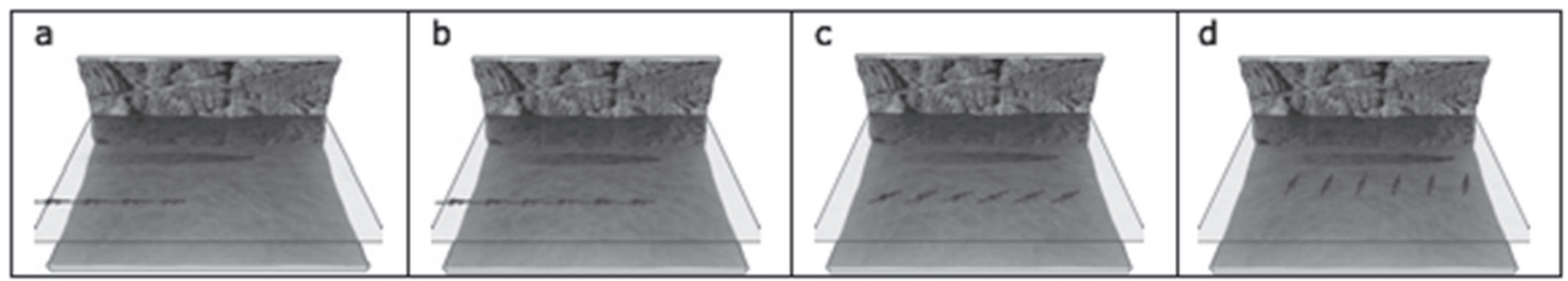

Estrategia en fila paralela a orilla sin vegetación con aguas someras.

Figura 5. Forrajeo en filas.

3. De corraleo: el encierro del cardumen que ejecuta el conjunto de individuos participantes en la práctica de caza (Figura. 6), con un $29 \%$ de recurrencia.

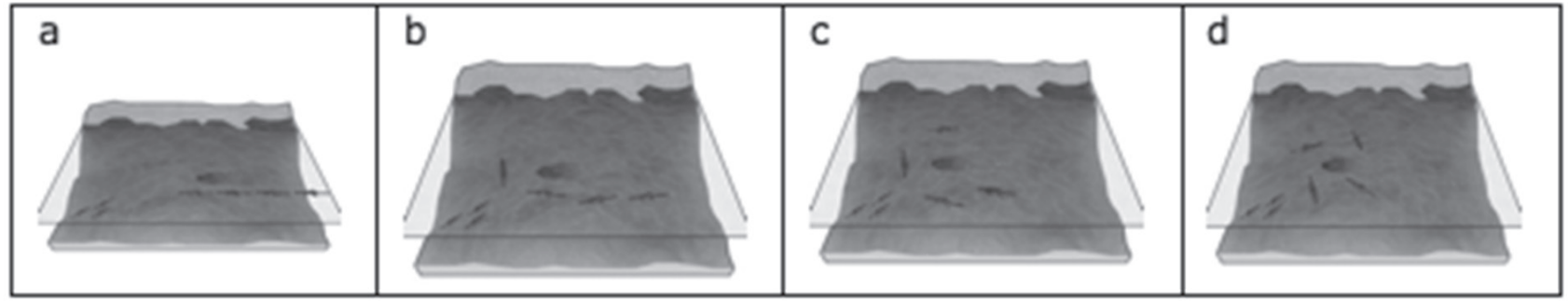

Cerco de cardumen en aguas abiertas.

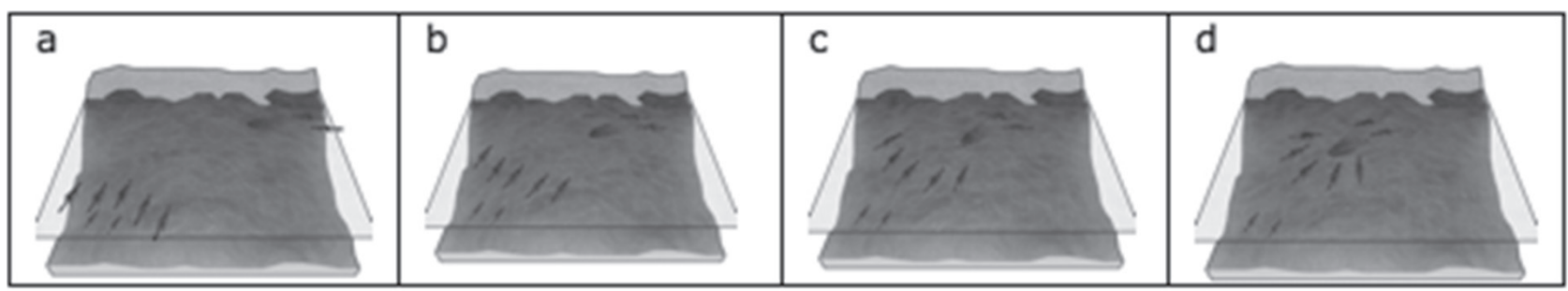

Corraleo

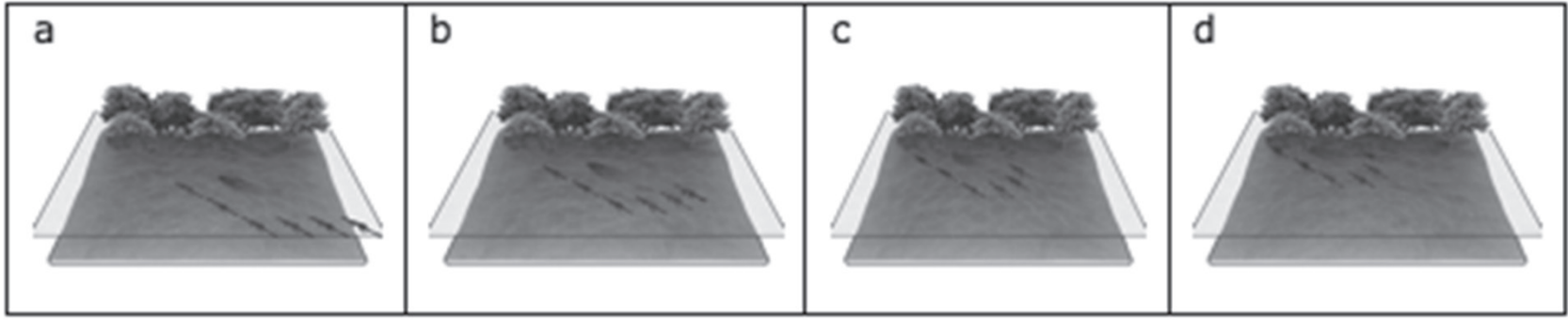


Corraleo en " $L$ "

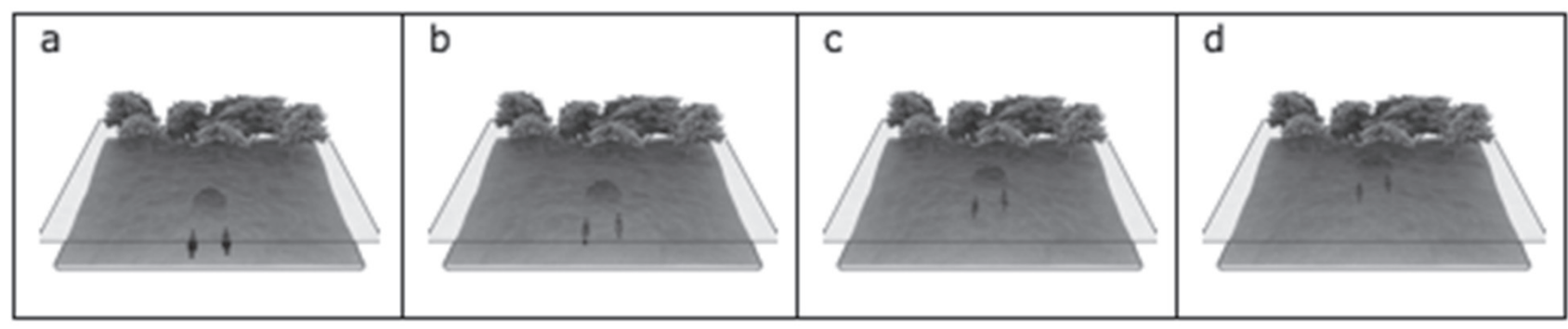

Pesca en pareja contra vegetación inundada

Figura 6. Forrajeo en Corraleo.

En cuanto a la captura cooperativa de peces, Staib (2005) señala que "la caza en grupo es característica de la nutria gigante" y estima que la distancia entre los integrantes de los grupos que cazan es generalmente de unos pocos metros. Al igual de lo observado por Hershkovitiz (citado por Duplaix, 1980), quien describe como un grupo de nutrias conduce peces a agua someras. Lo expresado por Staib y Hershkovitiz concuerda con los hallazgos del presente estudio, puesto que se identificaron y detallaron estrategias de caza para optimizar la captura, como se evidencia en algunas fotografías tomadas por otros autores en diferentes estudios confirman que las nutrias gigantes adoptan la formación " punta de flecha", tal es el caso de Duplaix en la fotografía No. 64 en la cual se observa la formación de punta de flecha en aguas abiertas (Groenendijk, Duplaix, Hajek, Schenck \& Staib, 2005, pág. 24).

Con respecto a las actividades cooperativas, se presentan debido a que las nutrias usan diferentes elementos para rodear el cardumen. Cuando se presenta la caza en aguas abiertas son los cuerpos de las nutrias, la barrera física que no permite dispersar el cardumen. Al desarrollarse caza contra la orilla o la vegetación, estas se presentan como barreras continuas y se confina el cardumen; además, la vegetación sumergida se presenta como una malla de caza que disminuye el gasto de la nutria para obtener alimento, razones por las cuales el tiempo de caza tiene una tendencia normal y simétrica explicado en el intervalo de 5 y 15 minutos.

Se evidencia como las nutrias gigantes adoptan comportamientos de caza que mitigan el esfuerzo y reducen al máximo el desgaste corporal para que su ejercicio de caza tenga mayor éxito (Kruuk, 2006) (Davenport, 2008) por consiguiente, un factor relevante que disminuye el gasto energético de las nutrias en la consecución de alimento.

Es importante resaltar que este fenómeno comportamental de las estrategias de caza de las nutrias surge evolutivamente debido a la especialización y organización de los peces en cardúmenes (Partridge, 1982). Lo anterior confirma que entre más grandes sea el grupo, mayor será el éxito de cacería.

Según las observaciones, las nutrias gigantes tienen una efectividad de caza individual (número de individuos que capturan peces / número total de nutrias) del $87 \%$ ( $n=222$ inmersiones durante el tiempo total de las observaciones) y una correlación lineal entre el número de individuos participantes en la alimentación y la efectividad de captura (Pearson's Product-Moment Correlation; Test: $\mathrm{t}=3,114, \mathrm{df}=43, \mathrm{p}=0,0001$ con correlación del 0,82 ) (Fig. 7). 


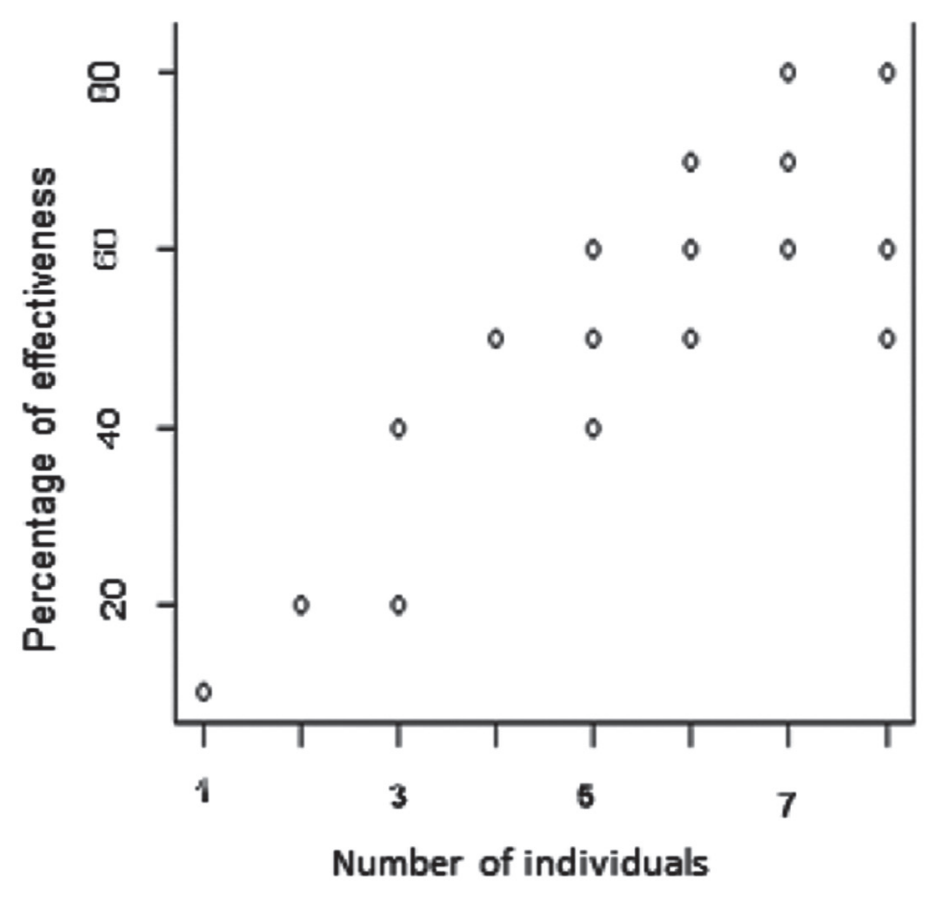

Figura 7. Efectividad de pesca Pteronura brasiliensis.

En cuanto a la efectividad de caza, se evidencia que un determinante en su realización es que se hace de manera cooperativa. No obstante, las actividades cooperativas para la consecución de alimento y actividades de cacería grupales no son exclusivos de $P$. brasiliensis. De igual forma, el desarrollo de estrategias de cacería, donde se cooperativiza el trabajo para la obtención de alimento, también es observado en nutrias euroasiáticas (Kruuk, 2006, pág. 156), carnívoros como leones (Stander \& Albon, 1963), lobos y perros africanos (Creel \& Creel, 1995). Con el desarrollo del trabajo cooperativo, estas especies demuestran una efectividad de hasta un $27 \%$ en felinos (Panthera leo) (Stander \& Albon, 1963, pág. 451) y en Licaones (Lycaon pictus) del $45 \%$ (Creel \& Creel, 1995, pág. 1330), calculo estimado por el éxito grupal en la captura. De acuerdo a lo observado se encontró que las nutrias gigantes tienen una efectividad de caza del $87 \%$.
El comportamiento de cooperación en actividades de forrajeo en nutrias gigantes es similar al de otras especies, hay que resaltar las diferencias. Una de ellas es el medio acuático en el que estos mustélidos consiguen su alimento, la asociación en cardúmenes por parte de sus presas como estrategia antipredadora y la individualidad en la captura final y consumo excepto para con las crías.

En el proceso de inmersión se analizaron el tiempo de inmersión y la profundidad (Figura. 8). Esta inmersión consistió en bucear, nadar para capturar peces y emerger con la presa y hacerlo en intervalos de tiempo que oscilaban de 6 a 35 segundos ( $n=222$ inmersiones) con $x=13$ segundos y $\sigma 2=8,54$, su distribución es simétrica, (Prueba de correlación productomomento de Pearson $\mathrm{p}=0,0032$ ). 


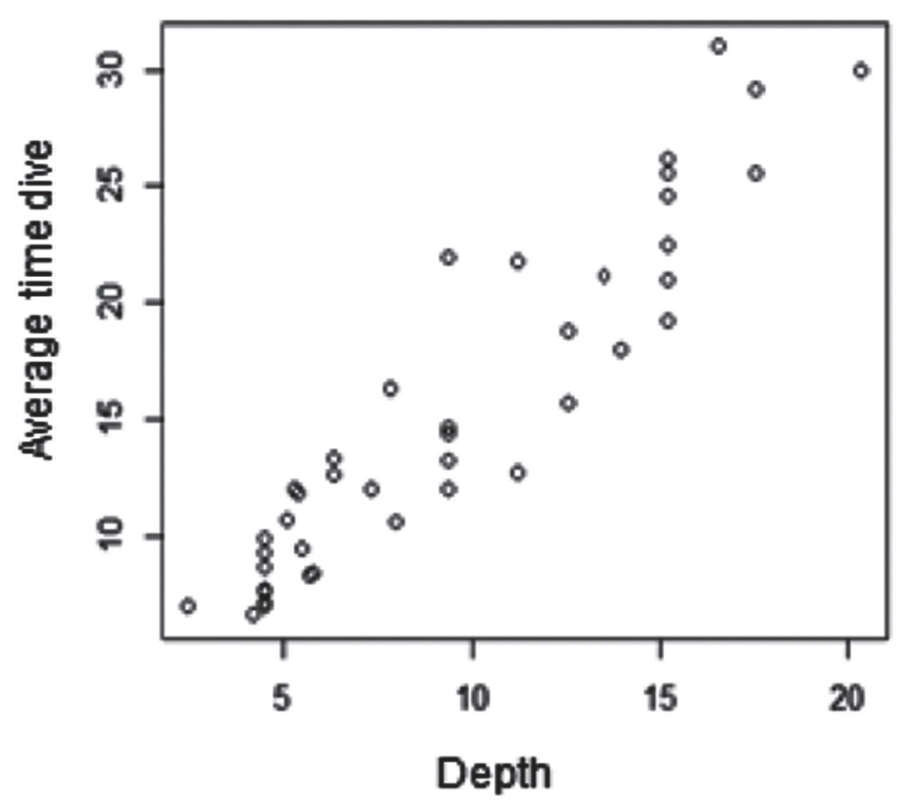

Figura 8. Relación lineal del tiempo de inmersión y profundad.

Para la caza cooperativa, las nutrias prefirieron cazar en tres tipos de áreas: aguas abiertas, vegetación sumergida y contra barranco; existiendo la asociación numérica (Chi-cuadrado: x $22=59,5024, p=0,0002$ ) entre el área de caza y estrategia empleada por la nutria gigante de la zona de estudio para la obtención de alimento.

Por otro lado, la época climática determina el comportamiento cooperativo de caza, observando que en verano (existe una menor área de dispersión del alimento), se presenta el fenómeno de caza cooperativa con una frecuencia del $55 \%$, encontrando un $p$-valor $=0,0576$ entre las variables tiempo atmosférico y tipo de orilla, con un nivel de confianza del $90 \%$. Esto se debe principalmente a dos fenómenos, el primero es la disminución del área del ecosistema acuático y el segundo, a la reorganización de los cardúmenes que posiblemente en invierno se escinden.

Estos dos fenómenos tienen una relación causal. Es decir, al existir un mayor confinamiento de los peces en un cuerpo de agua definido se organizan en cardúmenes como una estrategia anti predadora que permite ciertas ventajas: asegura una mejor protección contra los predadores (Partridge, 1982) al optimizar las alarmas colectivas, obligando a su predador, en este caso la nutria, a desarrollar comportamientos complejos de caza (Capman, 1990). Del mismo modo, al presentarse disminución del área del ecosistema acuático tiene consecuencias para la nutria y para los cardúmenes, la nutria tiene una mayor disponibilidad de peces confinados y los cardúmenes tienen menor área para maniobrar y escapar de los depredadores, por lo tanto, las nutrias prefieren bucear en áreas poco profundas, ya que producirán un bajo gasto de energía (Kruuk, 2006).

\section{CONCLUSIONES}

Por primera vez se reporta un comportamiento colectivo de caza para Pteronura brasiliensis. Este comportamiento, seguramente es una respuesta adaptativa a la cohesión en cardúmenes de sus presas y que se encuentra en un ecosistema abierto. Las estrategias de caza punta de flecha en nutria gigante de Santa Rosa de Sapuara demando un número efectivo de 7 individuos, los cuales permiten la optimización para la obtención de alimento en 
un $87 \%$ de eficiencia, resaltándose la importancia de la asociación entre las nutrias para optimizar la caza.

El tamaño de los grupos de nutria gigante es un elemento importante para tener en cuenta en los procesos de conservación de la especie y del complejo de humedales del Río Guaviare, como la Laguna de Santa Rosa de Sapuara. Ya que en estos se permiten el desarrollo de comportamientos alimenticios complejos y de ello inferir la salud de los especímenes que se encuentran en estos hábitats y el éxito de su recuperación para la zona como especie en peligro. Es importante destacar que los ecosistemas explotados de manera sostenible como el que llevan a cabo las comunidades indígenas y colonos presentes en los alrededores de la laguna permiten la subsistencia de la nutria y el hombre.

El comportamiento cooperativo en las actividades de pesca de la nutria gigante ( $P$. brasiliensis), permite evidenciar la organización de cooperación para obtener el alimento con una mayor eficiencia. En algunas actividades de pesca se evidenció la participación de los cachorros como proceso de aprendizaje.

Esta primera aproximación al comportamiento de Pteronura brasiliensis desde la perspectiva cooperativa de caza se convierte en un nuevo instrumento para el abordaje de estudio del grupo.

\section{AGRADECIMIENTOS}

A la fundación IASSOS por la financiación en especial a Jose Ávila, Alejandro Fontecha por la materialización de lo imaginado, Jorge Ariza por las ilustraciones, a Carlos Velandía y Lina Vásquez por su aporte; Carolina Corzo e Ivónne Salamanca por sus comentarios y revisión del escrito, Robinson Muñoz y a las comunidades Indígenas de Santa Rosa de Sapuara, etnias Piapoco, Puinabe y Sikuani, a todos los naturalistas que van más allá de los métodos.

\section{LITERATURA CITADA}

Alcock, J. (1993). Animal behavior: An evolutionary approach (Fifth ed.). Sunderland: Sinauer Associates. Recuperado de http://psycnet.apa.org/ psycinfo/1993-97586-000

Alexander, R. D. (1974). The Evolution of Social Behavior. Annual Review of Ecology and Systematics, V, 325-383. Recuperado el 28 de julio de 2015, de http://www.jstor.org/stable/2096892

Altmann, J. (1974, March 15). Observational Study Of Behaviour: Sampling Methods. Behaviour Magazine, XLIX, 227-265.

Álvarez-León, R. (2009, January-June). Importancia de los peces en la nutrición de la nutria gigante de rio (Pteronura brasiliensis) (carnívora: mustelidae) en Colombia. Luna Azul Journal(28), 8-14.

Arden-Clarke, C. H. (1986, June). Population density, home range size and spatial organization of the Cape clawless otter, Aonyx capensis, in a marine habitat. Journal of Zoology, CCIX(2), 201211. doi:10.1111/j.1469-7998.1986.tb03576.x

Blundell, G. M., Ben-David, M., \& Bowyer, T. (2002, May 14). Sociality in river otters: cooperative foraging or reproductive strategies? Behavioral Ecology, XIII(1), 134-141. Recuperado el 10 de febrero de 2015, de Behavioral Ecology Web Site: http://beheco.oxfordjournals.org/content/13/1/134.full.pdf+html

Blundell, G. M., Ben-David, M., Groves, P., Bowyer, R. T., \& Geffen, E. (2004, June 11). Kinship and sociality in coastal river otters: are they related? Behavioral Ecology, XV(5), 705-714. doi:10.1093/ beheco/arh110

Bohórquez González, A. (1994). Filogenia del Comportamiento. (L. García de Andrade, Ed.) Bogotá, Colombia: Universidad Incca de Colombia.

Capman, R. (1990). El animal y su universo, estudio dinamico del comportamiento (Primera ed.). (S. Fernández, Trans.) México, México: Fondo de la cultura económica.

Carrasquilla, M. C., \& Trujillo, F. (2004). Uso De Hábitat, Comportamiento Y Dieta De La Nutria Gigante (Pteronura brasiliensis) En El Rio Orinoco. Vichada - Colombia. En El Bajo Río Bita (Vichada-Colombia). En F. Trujillo, M. C. Diazgranados, L. Fuentes, Y. Llanos, J. Martínez, D. N. Castelblanco, .... G. Bravo, M. C. Diazgranados, \& F. Trujillo (Eds.), Estudios De Fauna Silvestre En Ecosistemas Acuáticos En La Orinoquía 
Colombiana (pp. 179 - 201). Bogotá, Colombia: Pontificia Universidad Javeriana, Facultad de Estudios Ambientales y Rurales.

Creel, S., \& Creel, N. (1995, March 14). Communal hunting and pack size in African wild dogs, Lycaon pictus. Animal Behaviour, L(5), 1325-1339.

Cutts, C. J., \& Speakman, J. R. (1994, January 12). Energy Savings In Formation Flight Of Pink-Footed. J. exp. Biol, CLXXXIX(1), 251-261. Recuperado el 2 de febrero de 2014, de http://jeb.biologists.org/content/jexbio/189/1/251.full.pdf

Davenport, L. (2008). Behavior and Ecology of the giant Otter (Pteronura brasiliensis) in Oxbow Lakes of the Manú Biosphere Reserve, Perú. North Carolina: The University of North Carolina at Chapel Hill.

Davenport, L. (2010, June 30). Aid to a Declining Matriarch in the Giant Otter (Pteronura brasiliensis). (K. McComb, Editor) doi:10.1371/journal. pone.0011385

Domínguez, C. A. (1985). Amazonía Colombiana, Visión General. Bogotá, Colombia: Talleres Gráficos Banco Popular.

Duplaix, N. (1980). Observations on the ecology and behavior of the Giant River Otter Pteronura brasiliensis in Suriname. Revue d'Écologie Terre et Vie., XXXIV(4), 495-620.

Evangelista, E. (2004, August 18). Change Of Partners In A Giant Otter Alpha Couple. IUCN Otter Spec. Group Bull., XXI(1), 47-51.

Evangelista, E., \& Rosas, F. (2011, March). The Home Range And Movements Of Giant Otters (Pteronura brasiliensis) In The Xixuaú Reserve, Roraima, Brazil. IUCN Otter Spec, I(A)(28), 31-37.

Gittleman, J. L. (1989). Carnivore Group Living: Comparative Trends. In Cornell University Press, \& J. L. Gittleman (Ed.), Carnivore Behavior, Ecology, and Evolution (First ed., pp. 183-207). New York, USA: Chapman \& Hall. doi:10.1007/978-14613-0855-3_8

Gómez, j. R. (2004). Ecología alimentaria de la nutría gigante (Pteronura brasiliensis) en el bajo Río Bita (Vichada-colombia). En f. Trujillo, m. C. Diazgranados, I. Fuentes, y. Llanos, j. Martínez, d. N. Castelblanco, .... G. Bravo, m. C. Diazgranados, \& f. Trujillo (eds.), Estudios de fauna silvestre en ecosistemas acuáticos en la orinoquía colombiana (p. 403). Bogotá, colombia: Pontificia Universidad Javeriana, Facultad de Estudios
Ambientales y Rurales, Instituto de Estudios Ambientales para el Desarrollo, Departamento de Ecología y Territorio.

Groenendijk, J., Duplaix, N., Hajek, F., Schenck, C., \& Staib, E. (2005). Standard Field Survey Techniques for the Giant Otter. En J. Groenendijk, F. Hajek, N. Duplaix, C. Reuther, P. Van Damme, C. Schenck, ... J. C. Botello, C. Reuther, O. Kölsch, \& W. Janßen (Eds.), Habitad. Arbeitsberichte der Aktion Fischotterschutz (First ed., pp. 11-30). Hankensbüttel, Germany: Verlag der GN-Gruppe Naturschutz GmbH. Retrieved October 7.

Groenendijk, J., Duplaix, N., Marmontel, M., Van Damme, P., \& Schenck, C. (2015, June 2). Pteronura brasiliensis. Retrieved October 7, 2015, from The IUCN Red List of Threatened Species Web Site: http://www.iucnredlist.org/details/18711/0

IDEAM (2012). Valores totales diarios de precipitación. Estación 3215006 Sapuara, Municipio de Barrancominas, Departamento del Guainía. Instituto de Hidrología y Meteorología y Estudios Ambientales. Bogotá D.C.

Klopfer, P. (1976). Introducción al comportamiento animal: un siglo de Etología. (J. J. Utrilla, Trans.) México: Fondo de Cultura Económica.

Kruuk, H. (1995). Wild Otters. Predation and Populations. Oxford, Estados Unidos: Oxford University Press.

Kruuk, H. (2006). Otters. Ecology Behaviour and conservation (First ed.). New York, Estados Unidos: Oxford University press.

Kruuk, H., \& Moorhouse, A. (1991, May). The spatial organization of otters (Lutra lutra) in Shetland. Journal of Zoology, CCXXIV(1), 41-57. doi:10.1111/j.1469-7998.1991.tb04787.

Maier, R. (2001). Comportamiento Animal, un enfoque evolutivo y ecológico (First ed., Vol. I). (J. Cejudo, Ed., \& A. Perez, Trans.) Madrid, Spain: Mc Graw Hill.

Martin, P., \& Bateson, P. (1991). La medición del comportamiento. Madrid, España: Alianza Editorial.

Mason, C., \& Macdonald, S. (1986). Otters. Ecology And Conservation. London, England: Cambridge University Press.

Melquist, W. E., \& Hornocker, M. G. (1983, April). Ecology of River Otters in West Central Idaho. Wildlife Monographs(83), 3-60. Recuperado de http://www.jstor.org/stable/3830731 
Mumm, C., Urrutia, M., \& Knörnschild, M. (2014, February). Vocal individuality in cohesion calls of giant otters, Pteronura brasiliensis. Animal Behaviour(88), 243-252. doi:10.1016/j.anbehav.2013.12.005

Partridge, B. L. (1982, August). Structure and function of fish schools. American Scientific, CCXLVI(6), 114-123.

Pérez Eslava, H., Bucheli León, P. E., \& Giraldo Benavides, B. (2005). La agroforestería en Guainía: Una alternativa sostenible. Instituto Amazónico de Investigaciones Científicas, SINCHI, Amazónas. Leticia: Equilátero.

Portocarrero, M., Morales, D., Díaz, D. L., \& Millán, J. P. (2009). Nutrias de Colombia. Bogotá: Fundación Omacha-Fundación Horizonte Verde.

Rudas, A., \& Prieto, A. (1998). Caracterización Ecológica Preliminar de las riberas del río Inírida (Guainía), en el área de influencia de la comunidad de La Ceiba. Bogotá: Universidad Nacional de Colombia, Facultad de Ciencias, Instituto de Ciencias Naturales.

Staib, E. (2005). Eco-Etología del Lobo de Rio (Vol. 1). (R. Duarte, Trans.) Lima, Peru: Sociedad Zoológica de Francfort.
Stander, P., \& Albon, S. (1963). Hunting success of lions in a semi-arid environment. Simposio de la Zoological Society of London(65), pp. 127 - 143.

Trujillo, F., Botello, J. C., \& Carrasquilla, M. C. (2006). Perro de agua, Pteronura brasiliensis (Orden Carnivora - Familia Mustelidae). En J. Rodríguez, F. Alberico, J. Trujillo González, \& J. Jorgenson, Libro rojo de los mamíferos de Colombia. Serie Libros Rojos de Especies Amenazadas de Colombia. (pp. 133-138). Bogotá, Colombia: Ministerio de Ambiente, Vivienda y Desarrollo Territorial.

Velandia, C., \& Vásquez, L. (2015). Estudio de La ecología alimentaria de Pteronura brasiliensis, mediante la investigación acción I-A como estrategia de conservación en la laguna Santa Rosa De Sapuara, Departamento de Guainía (Orinoquía Colombiana). Undergraduate Thesis, Universidad Distrital Francisco José De Caldas., Bogotá.

Whiten, A., Cusanse, D., Gómez, J., Teixidor, P., \& Bard, K. (1996). Imitative Learning of artificial fruit processing in children (Homo sapiens) and chimpanzees (Pan troglodytes). Journal of Comparative Psychology(110), 3-14.

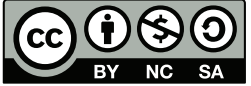

